\title{
A case report of renal calyceal diverticulum with hypertension in children and review of literature
}

\author{
Yongxiang Zhao ${ }^{1 \dagger}$, Ruimin Zhang ${ }^{1 \dagger}$, Ye Yun ${ }^{1}$, Xiangming Wu' ${ }^{1}$, Haowei $\mathrm{Li}^{1}$, Jun Wang ${ }^{1}$, Wei Wang ${ }^{1}$, \\ Chunmei Jia ${ }^{1^{*}}$ and Hongcheng Song ${ }^{2^{*}}$ (D)
}

\begin{abstract}
Background: Renal calyx diverticulum refers to a cystic lesion covered with the transitional epithelium in the renal parenchyma. Although there is no clear evidence that calyx diverticulum can cause hypertension, there exists a close association between the two, and there are few related reports. Herein, we reported the case of a child with renal calyx diverticulum complicated with hypertension and summarized the diagnosis and treatment.
\end{abstract}

Case presentation: Physical examination of the patient, an 11-year-old child, revealed a left renal cyst with hypertension $(155 / 116 \mathrm{mmHg})$. There were no related symptoms. Routine urine and blood biochemical examinations showed no abnormalities. Imaging revealed left renal cyst compression causing the hypertension. She underwent renal cyst fluid aspiration and injection of a sclerosing agent into the capsule, but her blood pressure increased again 3 days postoperatively. Color Doppler ultrasonography showed that the size of the left renal cyst was the same as that preoperatively. To further confirm the diagnosis, cystoscopic retrograde ureteropyelography was performed to confirm the diagnosis of renal calyx diverticulum. Subsequently, renal calyceal diverticulum resection and calyx neck enlargement were performed. The operation went smoothly and the blood pressure returned to normal postoperatively. No abnormalities were noted at the 7-month postoperative follow-up.

Conclusion: There exists an association between renal calyx diverticulum and hypertension. Therefore, hypertension can be considered a surgical indication for renal calyx diverticulum. Moreover, renal calyceal diverticulum in children can be easily misdiagnosed as a renal cyst. Therefore, it is important to be vigilant to prevent a series of complications, such as postoperative urine leakage, in such cases.

Keywords: Children, Calyceal diverticulum, Renal cyst, Hypertension

\section{Background}

Renal calyceal diverticulum refers to a cystic lesion covered with the transitional epithelium in the renal parenchyma [1], which is connected to the calyceal or renal pelvis through a narrow passage. It is difficult to differentiate between this lesion, the renal pelvis, and paracal cystic diseases [2], which could easily lead to misdiagnosis. Presently, reports on hypertension caused by renal calyceal diverticulum in children are rare. Here we report a case of renal calyceal diverticulum with hypertension in The Fourth Hospital of Baotou.

\footnotetext{
*Correspondence: jiachunmei_@sina.com; songhch1975@126.com

†Yongxiang Zhao and Ruimin Zhang are co-first authors and contributed equally to this study.

${ }^{1}$ The Fourth Hospital of Baotou, Baotou, Inner Mongolia, China

${ }^{2}$ Beijing Children's Hospital, Beijing, China
} 


\section{Case presentation}

This study was approved by the ethics committee of The Fourth Hospital of Baotou (ethics approval number is not applicable for our ethics committee). Written informed consent was obtained from the patient's parents.

The patient was an 11-year-old girl whose physical examination revealed left renal cyst with hypertension (blood pressure, 155/116 $\mathrm{mmHg}$ ). She had no back pain and no other symptoms such as frequent, acute, or painful urination. Her blood pressure upon admission was 153/113 mmHg. Oral amlodipine besylate (benzenesulfonic acid amlodipine) was used to normalize the blood pressure, following which routine urine and blood biochemical tests were performed. Urological CT revealed normal size, shape, and location of both kidneys. The left kidney was round, had a clear outline, had low density, and measured about $47.2 \times 46.1 \times 59.3 \mathrm{~mm}$ in size, with clear boundaries on CT at approximately $6 \mathrm{HU}$. No significant enhancement was noted in enhanced CT for any of the phases. There was also no abnormal density in the fatty capsule surrounding both kidneys, no separation of the bilateral assembly system, and no thickening sign in the perirenal fascia (Fig. 1). Upon CTA of both renal arteries, both arteries were found to be of normal shape at their origins. There was no obvious stenosis and expansion. The left renal vein was normal and there was no obvious compression stenosis. Serum renin, serum angiotensin, serum aldosterone, thyroid function, plasma cortisol, and blood catecholamine levels were normal.

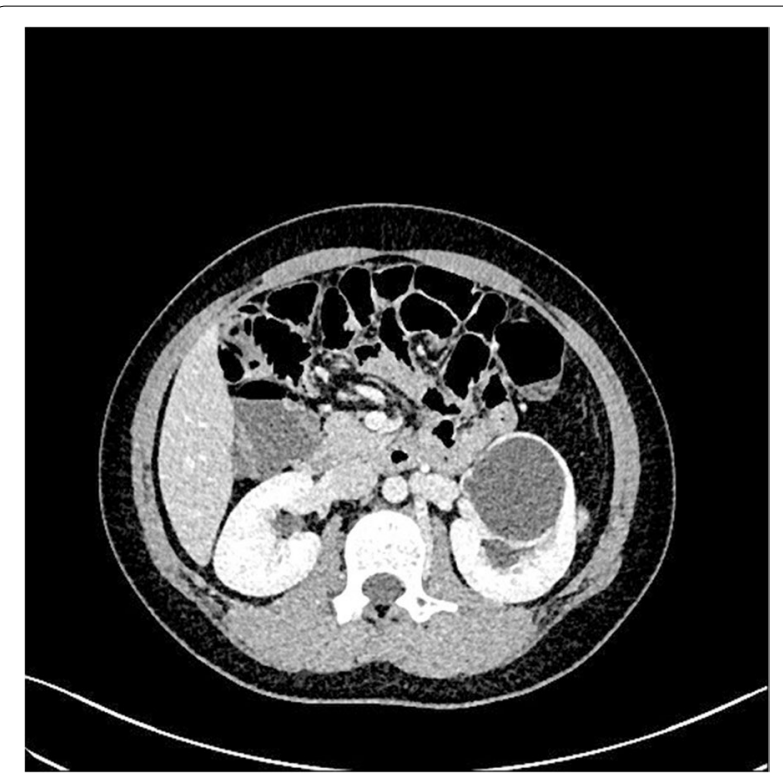

Fig. 1 Preoperative urological CT. A rounded hypointense shadow (approximately $47.2 \times 46.1 \times 59.3 \mathrm{~mm}$ ) with clear borders observed in the left kidney
Hypertension due to compression of the renal cyst was considered on the basis of imaging. She was admitted to the hospital and was monitored using color ultrasonography. Renal cyst fluid aspiration was performed, followed by intracapsular injection of a sclerosing agent. Her blood pressure returned to normal, but increased again on the third day. Repeat ultrasonography showed that the left renal cyst had returned to its pre-aspiration size. Hence, we noted the following: 1 . The injection of a sclerosing agent in the renal cyst is ineffective in children; soon after aspiration, the cyst fills again; hence, it is important to be vigilant for renal calyx diverticulum. 2. Hypertension in children is associated with cysts. Cystoscopic retrograde ureteropyelography was performed to further clarify the diagnosis. After anesthesia, cystoscopic retrograde intubation of the left ureter showed normal left upper and lower renal calyces and a spherically dilated middle calyceal contrast entry with a cyst attached to the renal pelvis (Fig. 2). The diagnosis of diverticulum of the renal calyces was confirmed. Open resection of the calyx diverticulum and enlargement of the calyx neck were performed. Upon intraoperative incision of the thin diverticular wall of the renal calyces, the diverticulum was observed to originate from the middle calyces of the kidney and the neck of the calyces was apparently narrowed. The narrowed calyces were enlarged to a diameter of approximately $1 \mathrm{~cm}$ by probing, and the incision was sutured continuously with locked edges to prevent restenosis. Subsequently, most of the dilated diverticular wall was excised and the thicker residual part of the calyx wall was closed. The 8-Fr catheter was left in the calyx neck as a stent tube, and the operation went smoothly. The patient's blood pressure decreased to normal on the postoperative day without the use of oral antihypertensive

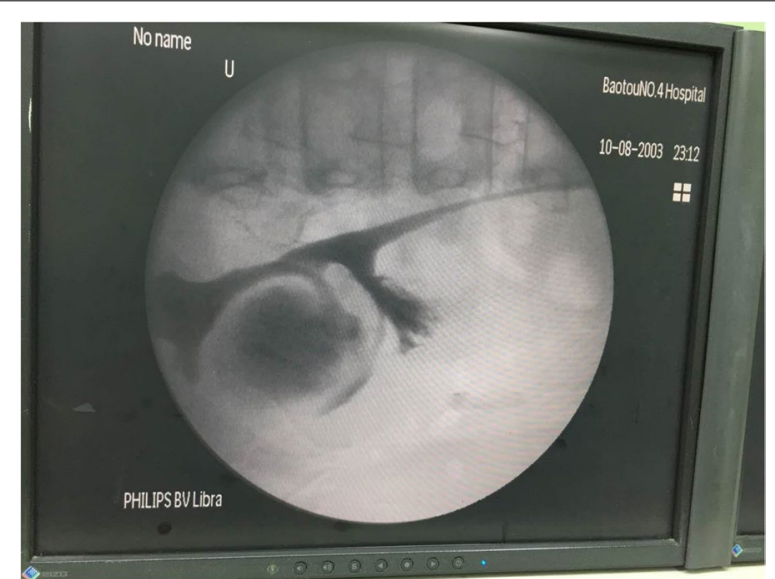

Fig. 2 Intraoperative C-arm view. The left superior and inferior calyces were normal, the middle calyces were spherically dilated, and the diverticulum was attached to the renal pelvis 
drugs, and the stent tube was removed 1 week postoperatively. At the 7-month postoperative follow-up and urological CT (Fig. 3), the patient showed good recovery, with no blood pressure abnormalities since discharge.

\section{Literature review}

The PubMed database was searched using the search term "calyceal diverticulum" for articles published between January 2010 and December 2020. A total of 36 patients with calyceal diverticulum were reported in the literature, which, combined with this report, makes 37 cases. The average age of the patients was 21.2 years (1-82 years), and the average age of the children was 8.5 years ( $1-17$ years) in 21 cases. There were four cases with no symptoms. No cases of hypertension in combination with renal diverticulum were reported. Fifteen cases were treated surgically, as described in Table 1 [3$20]$. Two of these cases could not be treated owing to the severity of the disease.

\section{Discussion and conclusion}

The prevalence of hypertension in children in China is $14.5 \%$ and is higher in males $(16.1 \%)$ than in females (12.9\%) [21]. Hypertension in early childhood often has no obvious symptoms. Common causes of this condition include congenital aortic stenosis, congenital renal hypoplasia, congenital urinary tract malformations, renal artery stenosis, latent glomerulonephritis, and adrenal disease. There are very few reports stating that hypertension in childhood is caused by renal calyceal diverticulum. Diverticula of the renal calyces in children is clinically rare, with a documented incidence of $0.6 \%$ [22]. A renal calyceal diverticulum is a sac-like structure that is located in the renal parenchyma and connected to the renal calyx. According to the different connection positions of the passage, it can be divided into type I and type II diverticulum. The former is connected to the minor renal calyx, mostly at one pole of the kidney, and the latter is connected to the major renal calyx, mostly at the central part of the kidney [23].

Secondary stones are more common in cases of calvarial diverticula, with a reported clinical incidence of 9.5-50.0\% among cases of calvarial diverticula caused by outflow tract obstruction and urinary reflux [24]. In the last decade, 10 of 36 cases of renal calyx diverticulum were complicated by stones. Further, among other clinical manifestations, pain was the most common symptom (17/36), and concomitant symptoms, such as fever (8/36) or bladder irritation, were often present when secondary urinary tract infection was present. In children, abdominal pain was the most common symptom (6/21). Four children had a combination of stones, and the diverticulum was rarely diagnosed in children. Considering that it resembles other cystic lesions of the kidney, further evaluation of children with renal cysts should be done in the presence of back pain, recurrent urinary tract infections, hematuria, and stones [20]. Per our experience, this disease may be misdiagnosed as renal cysts using ultrasonography and plain or even enhanced CT. Therefore, delayed contrast or delayed enhanced CT should be performed when the diagnosis is not confirmed or when renal calyx diverticulum is suspected. In addition, retrograde urography may also be performed to clarify the diagnosis. This CT + pyelography approach was used to confirm the diagnosis in 16 of 36 cases of renal calyx diverticulum. In cases for which imaging is not possible, cyst fluid aspiration can be performed under ultrasoundguided localization to assist in the diagnosis based on the cyst fluid composition [3], which has not been reported in the literature in the last decade.

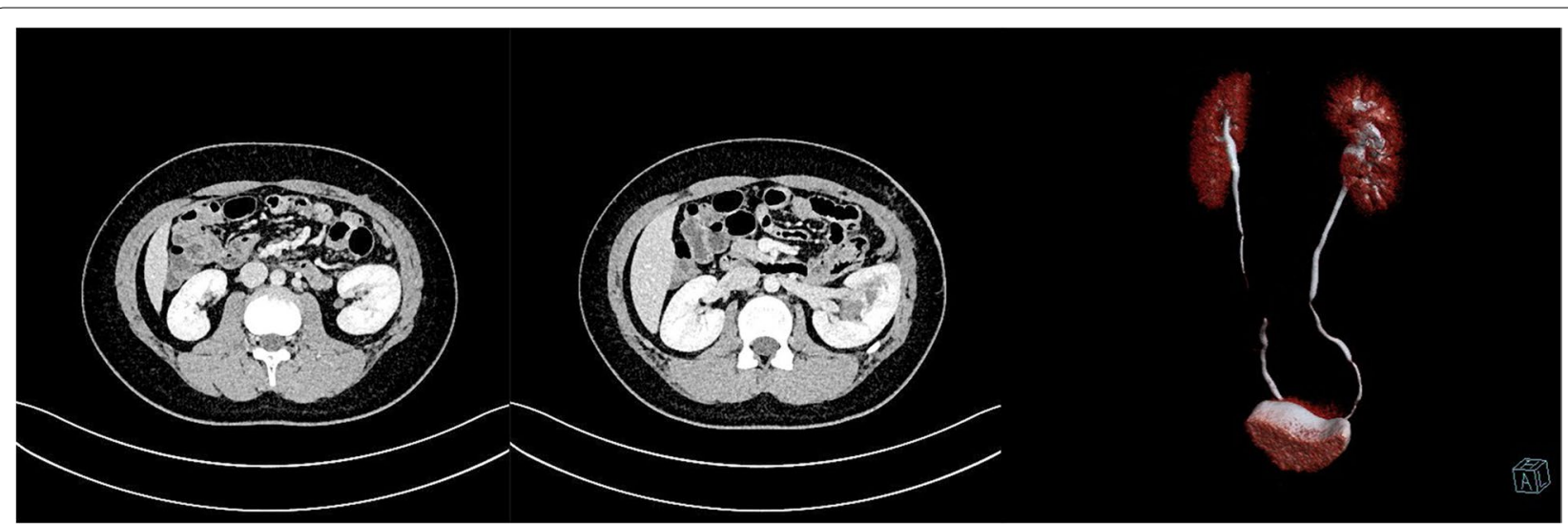

Fig. 3 Seven-month postoperative urological CT. The left kidney was slightly larger, with irregular morphology of the superior parenchyma, slightly enlarged local renal pelvis, and normal renal structure, considering postoperative changes 


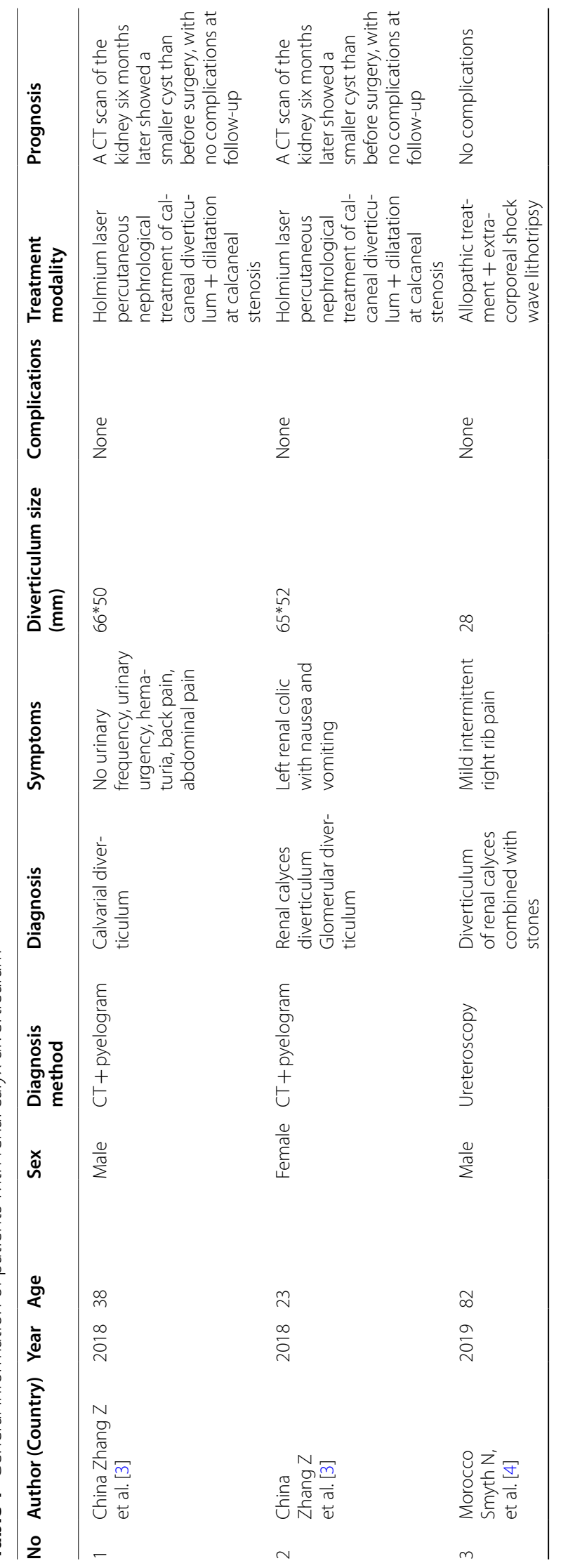




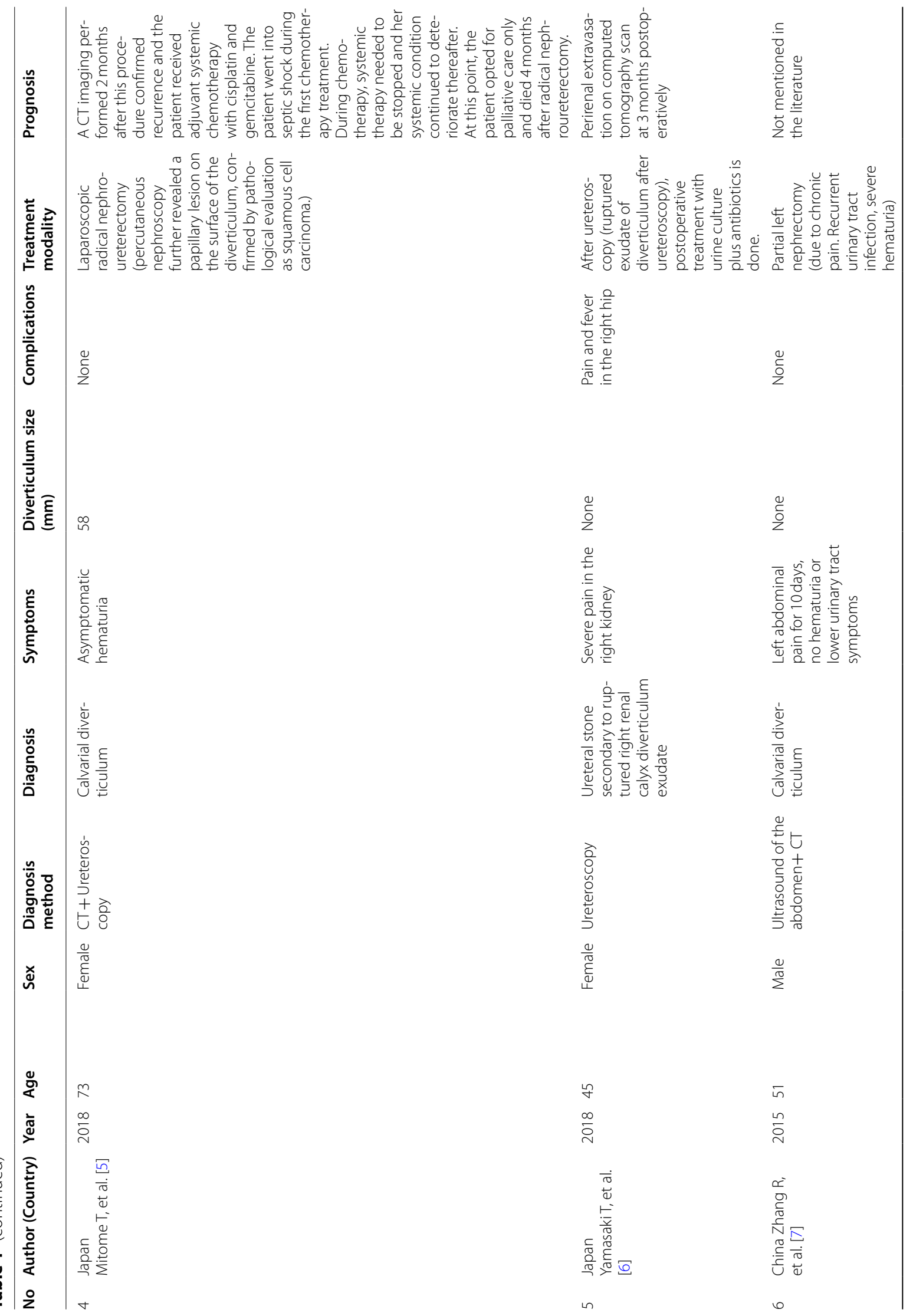




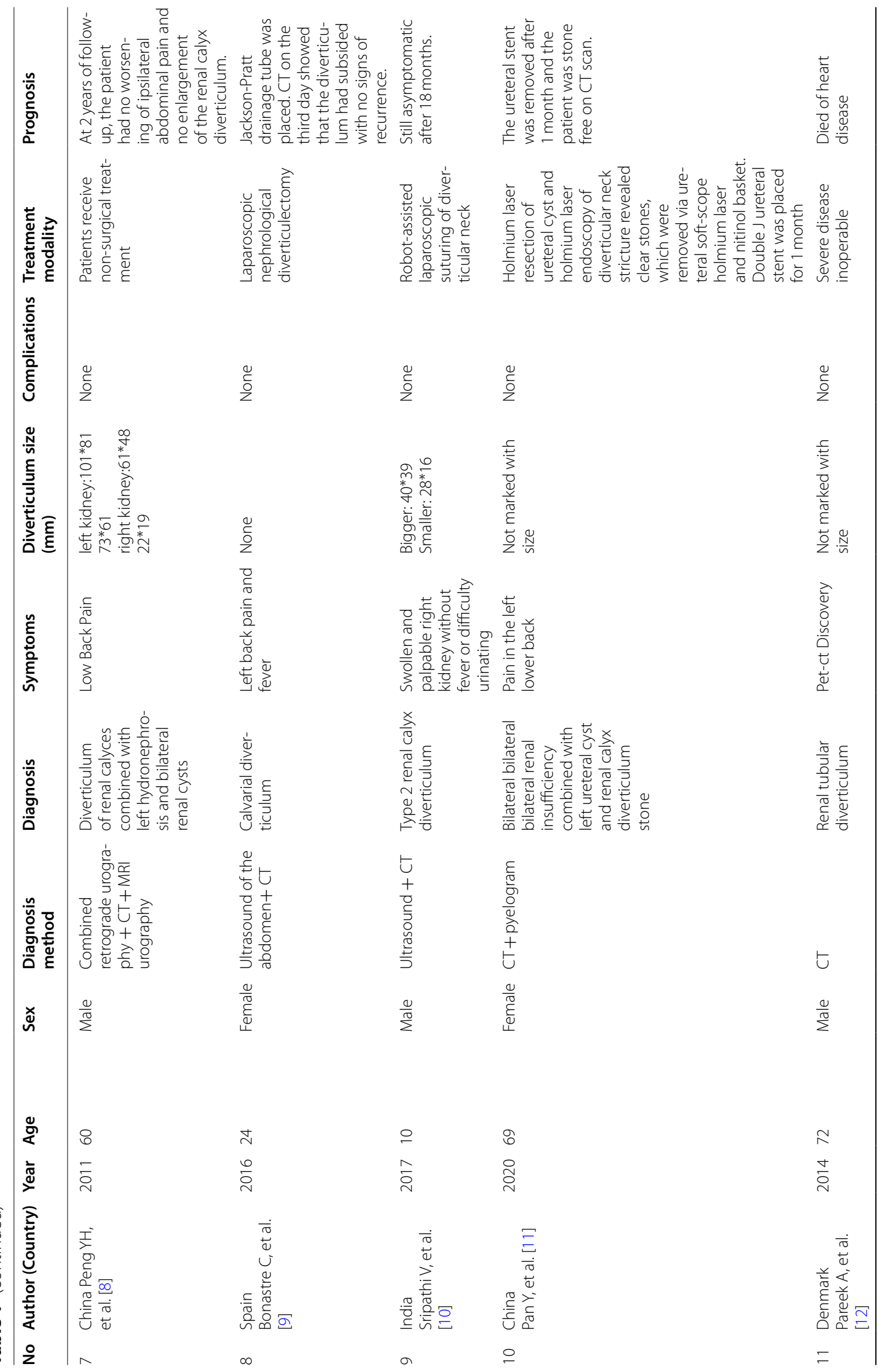




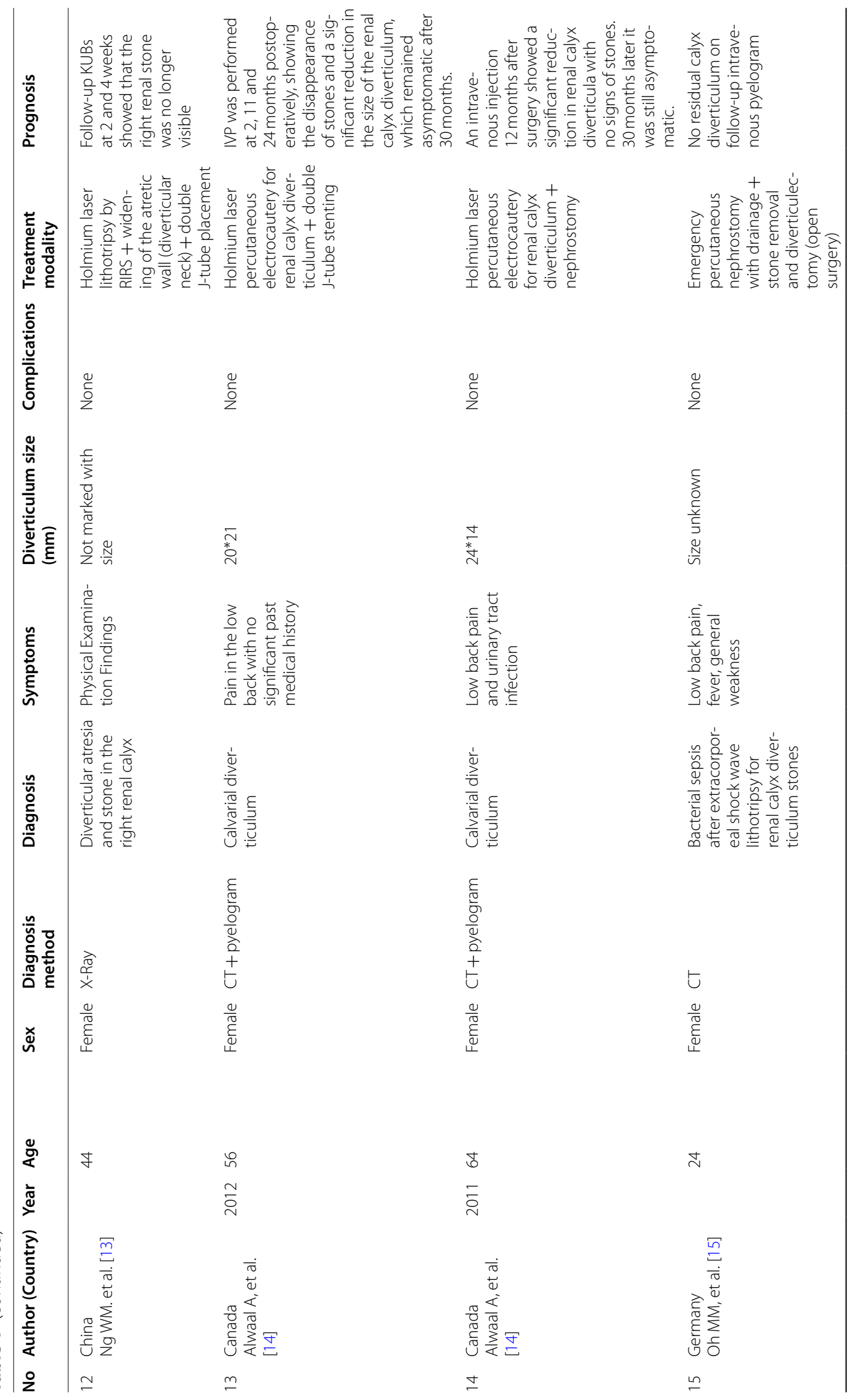




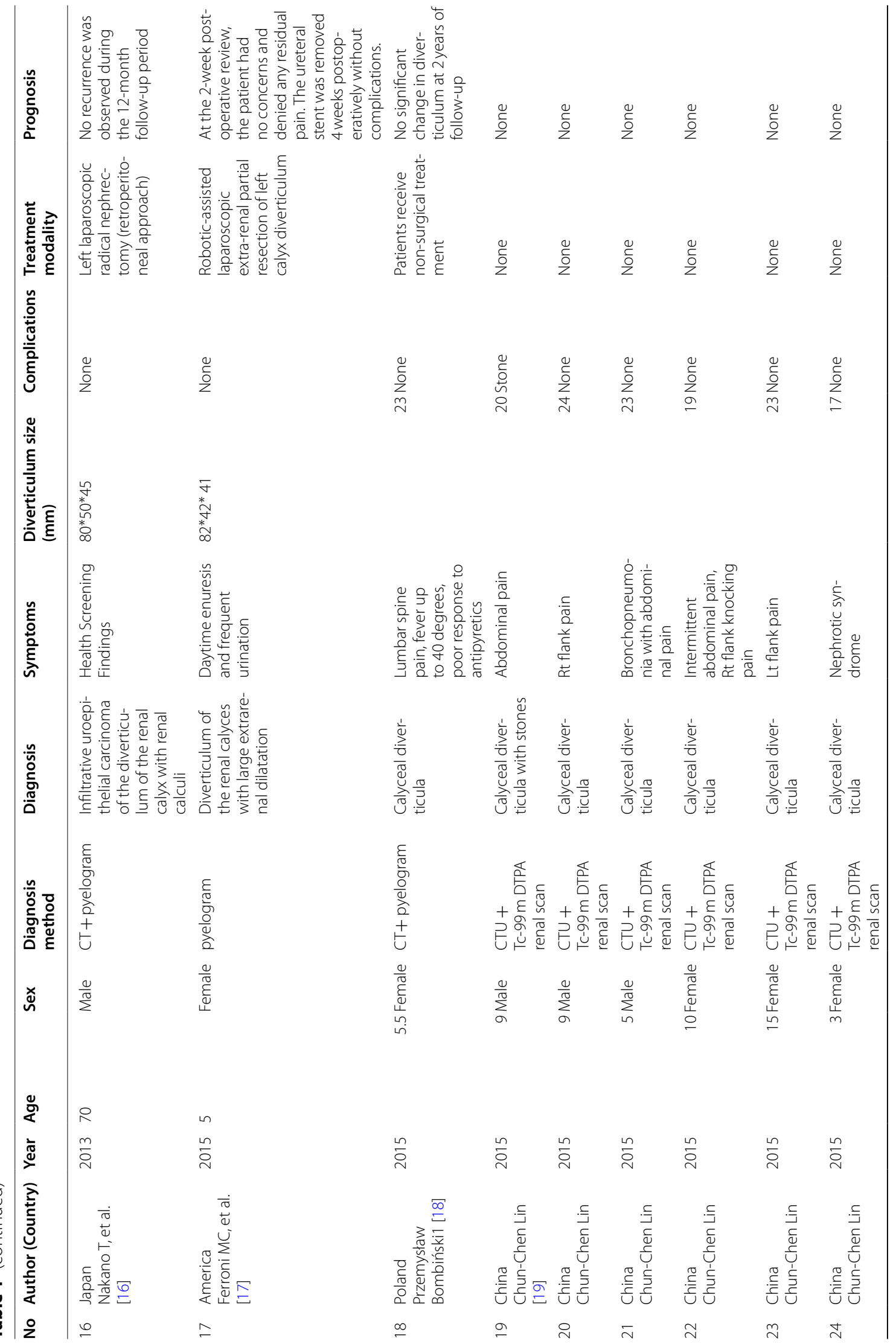




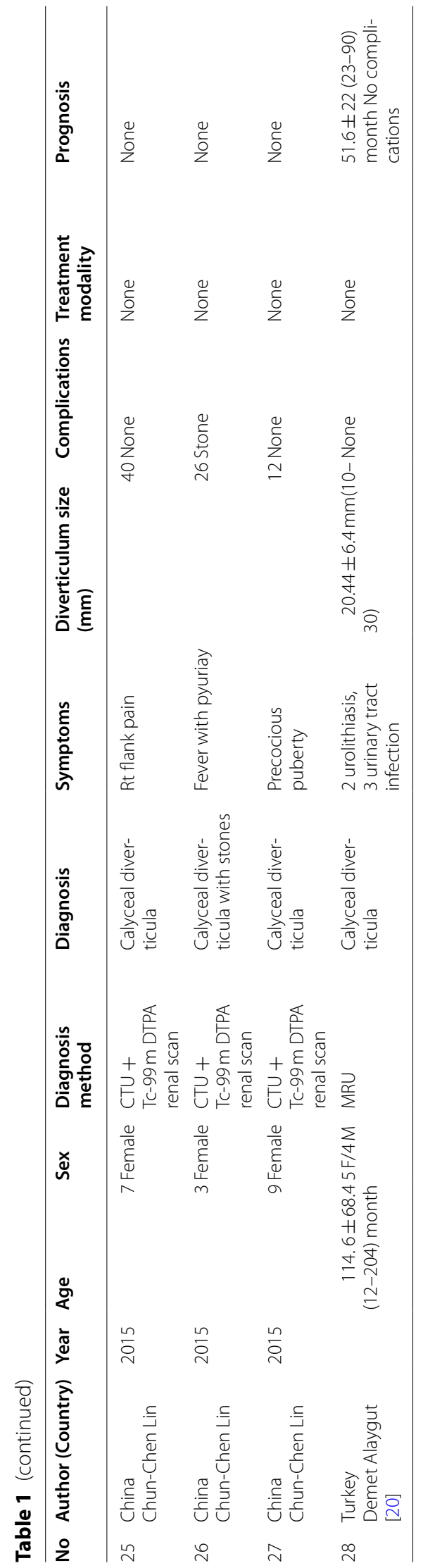


Renal calyceal diverticulum is not common among children, and only $20 \%$ of the cases eventually present symptoms [10]. In the last decade, 36 cases of renal calyx diverticulum were investigated, among which 21 were of children. Further, 15 patients were treated surgically for more obvious complications and 2 were treated symptomatically because they could not tolerate surgery. The treatment of renal calyx diverticulum needs to be determined by clinical symptoms. Symptomatic treatment can be given to children with no symptoms or mild clinical symptoms and for small-sized diverticula. Regular follow-up and surgery are recommended for children with large diverticula $(>4 \mathrm{~cm})$ or complications [1]. Kavukcu et al. [25] proposed that the treatment of the diverticulum depends on the complications, including repeated kidney infections, hematuria, and symptomatic kidney stones. Considering our experience, we believe that for children with hypertension and renal calyceal diverticulum, if the cause of hypertension cannot be determined, surgery should be performed in time. In our case, retrograde ureterography was performed preoperatively and a ureteral stent tube was left in place as a marker. The wall of the diverticulum was incised, the stenotic opening was found, the opening of the diverticulum was enlarged, the ureteral stent tube was visible and was determined to be connected to the renal pelvis, and the enlarged diverticulum opening was sutured with locked edges, with the stent tube left in place for support. The diverticulum wall was excised and the portion with the remaining renal tissue was sutured closed. The stent tube was removed 1 week postoperatively, and the patient's blood pressure returned to normal. The diverticulum did not recur on repeat ultrasonography and enhanced CT performed 7 months postoperatively, and the patient's blood pressure continued to remain normal.

Long et al. [26] suggested that ureteroscopy should be selected for middle and upper renal calyceal diverticula, whereas laparoscopic treatment should be considered first for larger exogenous lesions and lower pole diverticula. In well-equipped hospitals, robot-assisted technology can be used to help accurately identify and ligate the opening of the renal calyceal diverticulum to achieve better surgical outcomes [10, 27]. If diverticulum-induced stones are found in the preoperative examination, endoscopic percutaneous nephrolithotomy or laparoscopic treatment can be performed according to the location. However, ESWL is not ideal in the treatment of such stones $[28,29]$.

The diagnosis in this case was renal calyceal diverticulum with hypertension. Although there is no clear evidence stating that renal calyceal diverticulum can cause hypertension, there exists a close association between the two. During kidney transplantation, high blood pressure can also be "transplanted" along with the kidney, which sufficiently proves the important role of the kidney in blood pressure regulation. Any kidney disease could cause abnormal blood pressure [30-32]. Presently, the mechanisms by which kidney diseases can increase blood pressure mainly include sodium ion retention, renin-angiotensin system (RAS) dysregulation, sympathetic nervous system dysfunction, and endothelial cell-mediated vasodilation impairment [31]. In recent years, some scholars have discovered that the renal enzyme system is closely related to blood pressure [33], and its way of regulating blood pressure might be related to the metabolism of NADH [34] and catecholamines and the transport of sodium in the proximal renal tubules [35]. Malyszko et al. [36] pointed out that the mechanism underlying blood pressure regulation by renal enzymes and the relationship with dopamine receptors and endothelial function need further confirmatory evidence, and the specific mode of action is still unclear. Our patient had type II renal calyceal diverticulum with a large cyst. The cause of hypertension in this case could not be determined preoperatively, and the blood pressure immediately returned to normal postoperatively. Therefore, we considered the increase in blood pressure to be caused by cyst compression. The major reasons for this could be as follows: (1) Expansion of the cyst connected with the renal calyx compressed the sympathetic nerve branch, thereby causing constriction of the afferent arteriole of the corresponding nephron. (2) Compression caused renal ischemia and RAS activation. The formation of bladder cysts and long-term compression of the kidney might result in the loss of some nephrons and affect blood pressure regulation. (3) Abnormalities in the RAS might lead to abnormal cholesterol function, thereby affecting blood pressure regulation.

There exists an association between renal calyceal diverticulum and hypertension; hypertension can thus be a surgical indication in cases of renal calyceal diverticulum. In addition, renal calyceal diverticulum can be easily misdiagnosed as a renal cyst in children. Hence, it is important to be vigilant to prevent a series of complications, such as postoperative urine leakage, in such cases.

\section{Abbreviations}

CT: Computed tomography; CTA: Computed tomography angiography; RAS: Renin-angiotensin system; NADH: Nicotinamide adenine dinucleotide.

\section{Acknowledgements}

Not applicable.

\section{Authors' contributions}

YXZ designed the study and wrote the manuscript; RMZ and YY did the treatment plan and detected the therapy; XMW followed the patient; HWL, JW, and WW search the literature; CMJ designed the treatment plan; HCS designed the 
treatment plan and did the operation. All authors read and approved the final manuscript.

\section{Funding}

Not applicable.

\section{Availability of data and materials}

The datasets used and/or analysed during the current study are available from the corresponding author on reasonable request.

\section{Declarations}

\section{Ethics approval and consent to participate}

This study was approved by ethics committee of The Fourth Hospital of Baotou, and the number of ethics approval is not applicable in our ethics committee. Written informed consent was obtained from the patient's parents.

\section{Consent for publication}

Written informed consent was obtained from the patient's parents for publication of this Case report and any accompanying images. A copy of the written consent is available for review by the Editor of this journal.

\section{Competing interests}

The authors declare that they have no competing interests.

Received: 4 June 2021 Accepted: 17 December 2021

Published online: 11 January 2022

\section{References}

1. Koopman SG, Fuchs GJ. Intrarenal Stenosis and Stones: Calyceal diverticulum and infundibular Stenosis. In: Ureteroscopy edn: Springer; 2013. p. 407-16.

2. Sahin H, Sarioglu FC, Alaygut D, Akdogan Al, Pekcevik Y. Differentiation of simple renal parenchymal cyst and calyceal diverticulum. Pediatr Int. 2020;62:615-23.

3. Zhang Z, Zhang Y, Wang X, Chen D, Peng N, Chen J, et al. Challenges in the diagnosis of calyceal diverticulum: a report of two cases and review of the literature. J Xray Sci Technol. 2019;27:1155-67.

4. Smyth N, Somani B, Rai B, Aboumarzouk OM. Treatment options for Calyceal diverticula. Curr Urol Rep. 2019;20:37.

5. Mitome T, Tabei T, Tsuura Y, Kobayashi K. Squamous cell carcinoma in a Calyceal diverticulum detected by percutaneous Nephroscopic biopsy. Case Rep Oncol Med. 2018;2018:3508537.

6. Yamasaki T, Yoshioka T, Imoto M, Aoki H, Fujio K, Uehara S, et al. Rupture of a Calyceal diverticulum secondary to Ureteroscopy: a rare complication. Case Rep Urol. 2018;2018:9285671.

7. Zhang R, Shen W, Li X, Shen J, Tan J, Hao X. A Petal-like Calyceal Diverticulum. Urology. 2015;86:e31-2.

8. Peng YH, Zhang W, Gao XF, Sun YH. Calyceal diverticulum mimicking simple parapelvic cyst: a case report. Chin Med Sci J. 2015;30:56-8.

9. Bonastre C, Briones G. Laparoscopic calyceal diverticulectomy. Cent European J Urol. 2016;69:313.

10. Sripathi V, Mitra A, Padankatti RL, Ganesan T. Robotic treatment of a type 2 calyceal diverticulum in a child: is suture closure and marsupialisation enough for a good outcome? J Robot Surg. 2018;12:727-30.

11. Pan $Y$, Chen $\mathrm{G}$, Chen $\mathrm{H}$, Zhu Y, Chen $\mathrm{H}$. The left ureterocele and stone of calyceal diverticulum in the patient with bilateral incomplete duplex kidneys managed by flexible ureteroscopy: a case report and literature review. BMC Urol. 2020;20:35.

12. Pareek A, Laursen CB, Graumann O. Calyceal diverticulum: a benign imitator of serious pathology. BMJ Case Rep. 2014;2014:bcr2013202301.

13. Ng WM. Retrograde intrarenal surgery in atretic calyceal diverticular stone, a case report. Urol Case Rep. 2019;24:100840.

14. Alwaal A, Azhar RA, Andonian S. Percutaneous holmium laser fulguration of calyceal diverticula. Case Rep Urol. 2012;2012:716786.

15. Oh MM, Kim JW, Kim JW, Chae JY, Yoon CY, Park HS, et al. Bacterial sepsis after extracorporeal shock-wave lithotripsy (ESWL) of calyceal diverticular stone. Urolithiasis. 2013;41:95-7.
16. Nakano T, Kitagawa Y, Izumi K, Ikeda H, Namiki M. Invasive urothelial carcinoma within a calyceal diverticulum associated with renal stones: a case report. Oncol Lett. 2015;10:2439-41.

17. Ferroni MC, Rycyna KJ, Dwyer ME, Schneck FX. Calyceal diverticulum with a large extra-renal extension in a 5-year-old child. Urology. 2015;85:230-2.

18. Bombiński P, Warchoł S, Brzewski M, Biejat A, Dudek-Warchoł T, Krzemień $\mathrm{G}$, et al. Calyceal diverticulum of the kidney - diagnostic imaging dilemma in pediatric patients - case report. Pol J Radiol. 2015;80:27-30.

19. Lin CC, Shih BF, Shih SL, Tsai JD. Potential role of TC-99m DTPA diuretic renal scan in the diagnosis of Calyceal diverticulum in children. Medicine (Baltimore). 2015;94:e985.

20. Alaygut D, Şahin H, Çamlar SA, Soyaltın E, Öncel EP, Alparslan C, et al. Calyceal diverticulum of the kidney in pediatric patients - is it as rare as you might think? J Pediatr Urol. 2020;16:487.e1-6.

21. Dong B, Ma J, Wang HJ, Wang ZQ. The association of overweight and obesity with blood pressure among Chinese children and adolescents. Biomed Environ Sci. 2013;26:437-44.

22. Gross AJ, Fisher M. Management of stones in patients with anomalously sited kidneys. Curr Opin Urol. 2006;16:100-5.

23. Yu WW, Zhang DH, He X, Zhang YL, D. LG, Wang XL, Deng G, B. S, Wang YY. Incision and internal drainage treatment of renal cyst and calyceal diverticulum by endoscope. J Clin Urology. 2013;5:337-39+342.

24. Gandomi B, Bayat A, Kazemei T. Outcomes of septoplasty in young adults: the nasal obstruction Septoplasty effectiveness study. Am J Otolaryngol. 2010;31:189-92.

25. Kavukcu S, Cakmakci H, Babayigit A. Diagnosis of caliceal diverticulum in two pediatric patients: a comparison of sonography, CT, and urography. J Clin Ultrasound. 2003;31:218-21.

26. Long CJ, Weiss DA, Kolon TF, Srinivasan AK, Shukla AR. Pediatric calyceal diverticulum treatment: an experience with endoscopic and laparoscopic approaches. J Pediatr Urol. 2015;11(172):e1-6.

27. De Naeyer G, Schatteman P, Carpentier P, Mottrie A. 84 robot assisted laparoscopic calyceal diverticulum repair. Eur Urol Suppl. 2010;9:523-4.

28. Parkhomenko E, Tran T, Thai J, Blum K, Gupta M. Percutaneous Management of Stone Containing Calyceal Diverticula: associated factors and outcomes. J Urol. 2017;198:864-8.

29. Gross AJ, Herrmann TR. Management of stones in calyceal diverticulum Curr Opin Urol. 2007;17:136-40.

30. Mangray M, Vella JP. Hypertension after kidney transplant. Am J Kidney Dis. 2011;57:331-41.

31. Campese VM, Park J. The kidney and hypertension: over 70 years of research. J Nephrol. 2006;19:691-8.

32. Malyszko J, Malyszko JS, Mikhailidis DP, Rysz J, Zorawski M, Banach M. Hypertension and kidney disease: is renalase a new player or an innocent bystander? J Hypertens. 2012;30:457-62.

33. Desir GV, Peixoto AJ. Renalase in hypertension and kidney disease. Nephrol Dial Transplant. 2014;29:22-8.

34. Wu Y, Xu J, Velazquez H, Wang P, Li G, Liu D, et al. Renalase deficiency aggravates ischemic myocardial damage. Kidney Int. 2011;79:853-60.

35. Desir GV. Role of renalase in the regulation of blood pressure and the renal dopamine system. Curr Opin Nephrol Hypertens. 2011;20:31-6.

36. Malyszko J, Malyszko JS, Rysz J, Mysliwiec M, Tesar V, Levin-laina N, et al. Renalase, hypertension, and kidney - the discussion continues. Angiology. 2013;64:181-7.

\section{Publisher's Note}

Springer Nature remains neutral with regard to jurisdictional claims in published maps and institutional affiliations. 